\title{
COMPARATIVE ANALYSIS OF SOME MODAL RECONSTRUCTION METHODS OF THE SHAPE OF THE CORNEA FROM CORNEAL ELEVATION DATA
}

Andrei Martínez-Finkelshtein, Ph.D. (Corresponding Author)

Department of Statistics and Applied Mathematics, Almería University, 04120 Almería, Spain and Institute Carlos I of Theoretical and Computational Physics, Granada University, Spain

E-mail: andrei@ual.es

Antonia M. Delgado, Ph.D.

Department of Applied Mathematics, Granada University, Granada, Spain

E-mail: amdelgado@ugr.es

Gracia M. Castro, M.D. and Ph.D.

Ophthalmological Institute VISSUM-AImería, Almería, Spain

E-mail: graciacl@vissum.com

Alejandro Zarzo, Ph.D.

Department of Applied Mathematics, Madrid Polytechnic University, Madrid, Spain and Institute Carlos I of Theoretical and Computational Physics, Granada University, Spain E-mail: azarzo@gmail.com

Jorge L. Alió, M.D. and Ph.D.

Vissum Instituto Oftalmológico de Alicante (Department of Cornea and Refractive Surgery) and Division of Ophthalmology, Miguel Hernández University, Medical School, Alicante, Spain.

E-mail: jlalio@vissum.com

AMF and AMD are partially supported by Junta de Andalucía, grants FQM-229 and P06-FQM-01738. AMF and AZ are partially supported by the Ministry of Science and Innovation of Spain (project code MTM2008-06689-C02-01). AMD is also supported by the Spanish Ministry of Education and Science (project code MTM2006-13000-C03- 
02). Additionally, AMF is partly supported by Junta de Andalucía, grant FQM-481. All authors acknowledge also the support from the Carlos III Health Institute of the Ministry of Science and Innovation of Spain, grant PI08/90519. Last but not least, we are indebted to the anonymous referees, whose helpful comments clearly improved the text.

Word count: 4040 
Purpose. A comparative study of the ability of some modal schemes to reproduce corneal shapes of varying complexity is performed, using both standard radial polynomials and the radial basis functions (RBF). Our claim is that the correct approach in the case of highly irregular corneas should combine several bases.

Methods. Standard approaches of reconstruction by Zernike and other types of radial polynomials are compared with the discrete least squares fit (LSF) by the RBF in three theoretical surfaces, synthetically generated by computer algorithms in the lack of measurement noise. For the reconstruction by polynomials the maximal radial order 6 was chosen, which corresponds to the first 28 Zernike polynomials or the first 49 Bhatia-Wolf polynomials. The fit with the RBF has been carried out using a regular grid of centers.

Results. The quality of fit was assessed by computing for each surface the mean square errors (MSE) of the reconstruction by LSF, measured at the same nodes where the heights were collected. Another criterion of the fitting quality used was the accuracy in recovery of the Zernike coefficients, especially in the case of incomplete data.

Conclusions. The Zernike (and especially, the Bhatia-Wolf) polynomials constitute a reliable reconstruction method of a non-severely aberrated surface with a small surface regularity index (SRI). However, they fail to capture small deformations of the anterior surface of a synthetic cornea. The most promising is a combined approach that balances the robustness of the Zernike fit with the localization of the RBF.

\section{Introduction}

Zernike analysis is used commonly in ophthalmology to express ocular wavefront error in the form of a polynomial function ${ }^{1}$. The coefficients of these expansions have interpretation in terms of the basic aberrations such as defocus, astigmatism, coma, trefoil and spherical aberrations, along with higher order aberrations. As a fitting routine, Zernike polynomials are not limited to analysis of wavefront error surfaces, but can be applied to other ocular surfaces as well, including the anterior corneal surface $^{2,3}$. It has been suggested that Zernike analysis may be applicable towards the development of corneal topography diagnostic tools (e.g., Zernike coefficients as inputs into corneal classification neural networks ${ }^{4,5}$ ), replacing or supplementing the currently used corneal indices included with many topography devices. Given the significance of 
the shape of the front surface of the cornea to the refraction of the eye ${ }^{6}$ and the ability to correct refractive errors by laser ablation of the front surface of the cornea, a detailed wavefront error analysis of corneal topography data is clinically useful and important. It has been recognized that the corneal front surface generally provides the bulk of the ocular aberrations in the postsurgical or pathologic eye ${ }^{7}$.

However, several potential limitations of this approach have been reported in literature ${ }^{1,8}$. There is a growing concern that the Zernike fitting method itself maybe inaccurate in abnormal conditions. Furthermore, it is very difficult to assess a priori how many terms are necessary to achieve an acceptable accuracy in the Zernike reconstruction for any given corneal shape ${ }^{9}$. It is known ${ }^{8}$ that limiting Zernike analysis to only several orders may cause incorrect assessment of the severity of more advanced stages of keratoconus ${ }^{6}$. This information is particularly needed in the discriminant analysis of the decease markers, or when selecting the numerical inputs for neural network based diagnostic software such as corneal classification and condition severity grading utilities.

In this sense, several alternatives to Zernike polynomials have been recently suggested.

The purpose of this report is a comparative study of the ability of some modal approaches to reproduce corneal shapes of varying complexity. More than dwelling again on the shortcomings of the Zernike fit we compare several techniques in some "model" situations, ignoring on purpose all sources of noise that exist in any real system. In this study we avoid experiments using third-party software on corneal elevation from in vivo eyes, but implement the fitting methods on theoretical surfaces, synthetically generated by computer algorithms. This gives an insight into the intrinsic accuracy properties of each approach.

It should be emphasized that our primary goal was assessing the behavior of some methods in different situations. As a result of our study, we may conclude that there is no unique and best approach to cornea surface reconstruction that could be considered preferable in every scenario, so that a combination of techniques can be the optimal strategy.

\section{Modal reconstruction of a surface}

In corneal topography, the elevation of the corneal surface is collected on a discretely sampled grid, which is typically a polar grid for Placido-ring based systems. These raw data are used to reconstruct the corneal shape, by applying either zonal (see e.g. ${ }^{10}$ ) or modal algorithms. The modal approach is taken most often because it is easy to use 
and it offers better noise suppressing properties. Within the modal approach, the anterior surface of the cornea can be modeled by a linear combination of some basis functions,

$$
C(P)=\sum_{j=1}^{v} a_{j} f_{j}(P),
$$

where $C(P)$ is the corneal elevation at the point $P$ of the plane, $f_{j}$ are the a priori chosen basis functions, and $\mathbf{a}=\left(a_{1}, \ldots, a_{v}\right)^{T}$ are the expansion coefficients (the superindex $T$ here means matrix transposition). In this setting, fitting (1) to a discrete set of elevation data $\mathbf{Z}=\left(Z_{1}, \ldots, Z_{N}\right), \quad N \geq v$, at the nodes $P_{i}, i=1, \ldots, N$, can be restated in terms of solution of the overdetermined linear system

$$
M \mathbf{a} \approx \mathbf{Z}, \quad M=\left(f_{j}\left(P_{i}\right)\right)_{1 \leq i \leq N, 1 \leq j \leq v} .
$$

The least square fit (LSF) corresponds formally to the solution of the normal equations $M^{T} M \mathbf{a}=M^{T} \mathbf{Z}$, which is unique if the collocation matrix $M$ is of maximal rank $v$.

Different basis functions $f_{j}$ in (1) can be used, such as radial polynomials (Zernike, Bhatia-Wolf, etc.), Fourier series and radial basis functions (RBF).

Zernike polynomials $Z_{n}^{m}$ (corresponding to the radial $n \in \mathbb{N} \cup\{0\}$ and azimuthal $m \in \mathbb{Z}$ indices, with $|m| \leq n$ and $n-m$ even) exhibit special properties that make them an interesting expansion set for the description of general surfaces in the fields of optical engineering and in physiological optics. They form a complete set of orthonormal polynomials on the unit disc with respect to the Lebesgue (plane) measure. Since they are well-known, we omit their explicit description here, referring the reader to the standard ${ }^{11}$.

There are several methods to map the double indices $(n, m)$ into a $1-\mathrm{D}$ array $j$; the most widely acceptable one is

$$
j=\frac{n(n+2)+m}{2} .
$$

Choosing in (1) as $f_{j}$ the Zernike polynomials of radial order $\leq n$ yields the value $v=(n+1)(n+2) / 2$. Most clinical aberrometers use Zernike expansion up to the 6 th (typically, 4 th) radial order to reconstruct wavefront data or corneal surface ${ }^{12}$, corresponding to values $v=15$ and $v=28$ for $n=4$ and $n=6$, respectively. It was shown $\mathrm{in}^{9}$ that, at least for the normal and astigmatic corneas, the optimal value is $v=11$, so that even $n=4$ leads in most cases to over-parametrization of the model. 
Closely related with the Zernike polynomials are the Bhatia-Wolf polynomials ${ }^{13}, B_{n}^{m}$, whose fitting properties have been analyzed $\mathrm{in}^{14}$. They also satisfy the orthonormality condition with respect to the unit Lebesgue measure on the disc. One difference between Bhatia-Wolf and Zernike polynomials is that the only constraint $|m| \leq n$ on the radial and azimutal indices results in the generation of $(n+1)^{2}$ linearly independent polynomials for a given radial degree $n$, instead of the $(n+1)(n+2) / 2$ for the Zernike polynomials. It should be noted that $B_{n}^{m}$ are not algebraic polynomials in the Cartesian variables $x$ and $y$, but they expand in series of monomials $x^{i} y^{j} \rho^{k}, \rho=\left(x^{2}+y^{2}\right)^{1 / 2}$, for $i \geq 0, \quad j \geq 0$, and $k \geq 0$. The double indices $(n, m)$ are easily converted into the polynomial order $j$ of $B_{j}$ by $j=n(n+1)+m$.

There are other possible choices of radial polynomials, such as the generalized Zernike polynomials ${ }^{15}$ and the Sobolev orthogonal polynomials on the disk, see ${ }^{16,17}$.

A special remark deserves another well known fitting method based on the (bidimensional) Fourier transform ${ }^{18}$, which reconstructs wavefront data by decomposing the image into spatial frequency components (see e.g. ${ }^{19-22}$ ). Standard Fourier methods build the surface as a combination of the trigonometric basis whose coefficients can be computed via the FFT algorithm. In some situations the input information is the set of slopes and not elevations, in which case an additional step (reduction to the laplacian) is needed. The typical Stokes phenomenon (high oscillation at the boundary) is handled via a Gershberg-type iterative method (see the literature mentioned above).

In this paper, we investigate an alternative meshless technique for reconstructing the corneal shape from the elevation data using as $f_{j}$ in (1) sets of radial basis functions (RBFs), defined in their simplest form by translates of a given function $\Phi$ :

$$
f_{j}(\cdot)=\Phi\left(\left\|\cdot-Q_{j}\right\|^{2}\right),
$$

where points $Q_{j}$, called centers of the RBFs, are conveniently chosen, and $\|\cdot\|$ denotes the Euclidean distance on the plane. The general theory of interpolation by RBFs is developing fast, and several criteria for $\Phi$ can be found in the literature ${ }^{23}$. In particular, standard options are the so called Gaussians and Inverse multiquadrics, corresponding respectively to $\Phi(t)=\exp (-\alpha t)$ and $\Phi(t)=\left(t+c^{2}\right)^{-\beta}$, with positive parameters $\alpha, c$, and $\beta$. However, we are unaware of any deep theoretical analysis of the LSF with RBF. This, according to ${ }^{24}$, is a highly relevant and interesting field of research.

There are several advantages in the use of (1) with the RBF. Due to the fast decay 
of the Gaussians or multiquadrics, functions $f_{j}$ in (3) are practically locally supported. Hence, (1) exhibits features of the zonal approach, eventually capturing small deformations of the surface, which are missed by the polynomial fitting. The rate of decay or the size of the effective support of $f_{j}$ can be controlled with the parameters of the RBFs, endowing the model with a flexibility that lacks in other modal schemes described above. The correct selection of these parameters depends upon several factors, such as the frequency of the sampling data, the separation between centers of the RBFs and the grade of variation of the surface. As far as we are aware of, the only work where such a use of the Gaussians has been discussed, but in the context of the wavefront fitting, is ${ }^{25}$.

We want to point out that the choice of the RBF in (1) does not imply renouncing to the Zernike coefficients as the output information of the reconstructed surface. On the contrary, since the centers $Q_{j}$ are fixed a priori, the values of

$$
s_{m, n, j}=\frac{1}{\pi} \iint_{x^{2}+y^{2} \leq 1} Z_{n}^{m}(x, y) f_{j}(x, y) d x d y,
$$

with $f_{j}$ given by (3), can be previously computed and stored, so that the Zernike coefficients are easily recovered by scalar product of two vectors.

\section{Material and Methods}

It is well known that in a situation close to ideal, when the corneal surface presents only small and smooth deviations from a sphere,

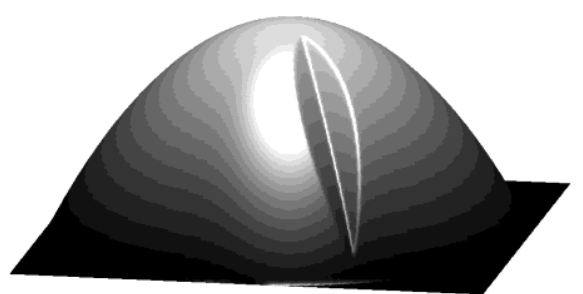

Figure 1: A 3D representation of surface $B$. almost any reasonable fitting scheme renders good results. In particular, in such a situation the use of the Zernike polynomials is perfectly justified. Hence, in order to assess the fitting properties of the different approaches, we have chosen the following three model surfaces with high surface regularity indices $(\mathrm{SRI})$ :

1. Surface A: a "flat sphere", roughly simulating an operated cornea and a surface with a gradient discontinuity (Fig. 4).

2. Surface B: a sphere with a radial deformation (“a scar", Fig. 1).

3. Surface C: a cornea with topographic asymmetry and decentered corneal apex (keratoconus), but with an incomplete set of data (Fig. 6). 
In cases $\mathrm{A}$ and $\mathrm{B}$ we obtain the elevation data at a discrete set of points $P_{s}$, $s=1, \ldots, 6144$, with polar coordinates $\left(\rho_{i}, \theta_{j}\right)$, where $\rho_{i}=i / 24, i=1, \ldots, 24$, while the meridians $\theta_{j}, j=1, \ldots, 256$, are equidistributed in $[0,2 \pi)$. For the surface $\mathrm{C}$ we collect the elevations at a subset of the nodes described above (see Fig. 2), simulating the standard situation in the clinical practice, when part of the measurements is obstructed by the eyelashes or other obstacles. A common procedure in such cases is to discard the elevations corresponding to incomplete rings, which may imply an unnecessary loss of information. One of the advantages of the RBF is that they are not bound intrinsically to circular domains. This fact gives an additional interest to the analysis of the situation modeled by Surface $C$.

Another important observation is related to the units of measurement. Since the elevations are obtained from synthetic surfaces where the scaling is irrelevant, we chose to fit the data on a unit disk. Hence, the plots appearing in the figures below are given in universal units, whose choice does not affect the results.

We gather the discrete elevation data into a vector $\mathbf{Z}$, without adding any noise, and solve the

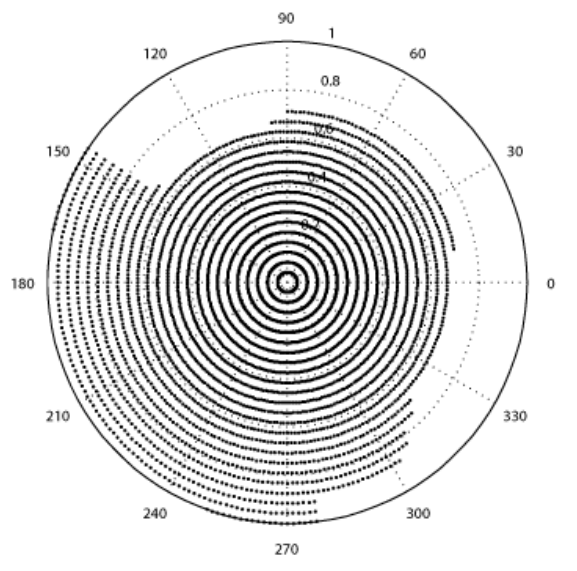

Figure 2: Elevation data for Surface C. overdetermined system (2) in the sense of the LSF. In practice, the collocation matrix $M$ can be very ill-conditioned and numerically rank deficient, so we have to avoid solving the normal equations $M^{T} M \mathbf{a}=M^{T} \mathbf{Z}$ directly. The use of the Moore-Penrose pseudoinverse of $M$ computed by its singular value decomposition (SVD), complemented with regularization, is preferable instead, see e.g. ${ }^{26}$.

The method can be easily adapted to include the weighted least square fit (WLSF) by left-multiplying equation (2) by a diagonal positive matrix representing the weights. In real-life computation, these weights can reflect the reliability of the data (portions of the cornea obstructed by eyelashes, poor quality of the tear film, etc.; see e.g. ${ }^{27}$ for the

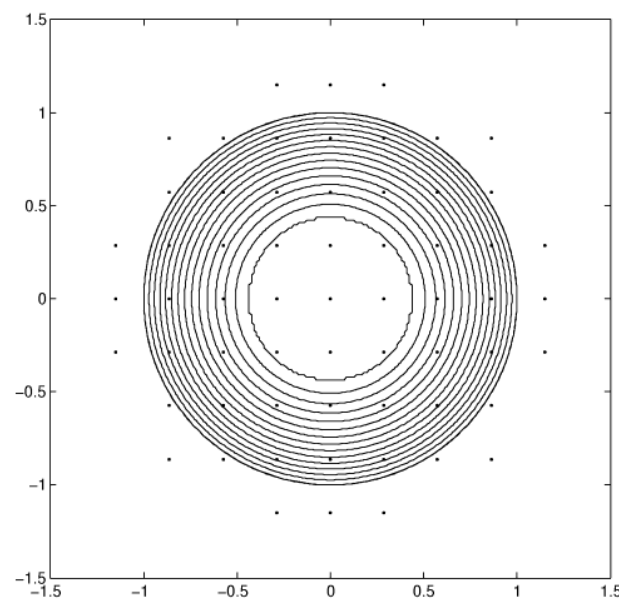

Figure 3. Dots denote centers of the RBF. 
algorithms that allows to separate and identify the regions of a strong interference).

All the computations have been carried out on a PC running version 7.6 of Matlab (MathWorks Inc.). The vectorization capabilities of Matlab have been intensively exploited, achieving highly efficient algorithms that reduced drastically the computation time to less than 3 seconds even for the most time-consuming Zernike polynomials fit (compare e.g. with ${ }^{1,3}$ ).

We have performed a comparison between families of radial polynomials (namely, Zernike and Bhatia-Wolf polynomials) and radial basis functions (Gaussians and inverse multiquadrics). For the reconstruction by polynomials we normally choose the maximal radial order 6 , which corresponds to the first 28 Zernike polynomials, or the first 49 Bhatia-Wolf polynomials, which is the standard in modern aberrometers ${ }^{12}$. The fit with the RBF has been performed using a regular grid of centers, like those represented in Fig. 3. Observe that in order to avoid high oscillations on the edge we must use centers situated outside of the cornea, although we omit those located too far from the nodes.

Experiments have been performed also with other functions, such as Sobolev orthogonal polynomials on the disk or multiquadric RBF; however, the results obtained do not differ significantly from those corresponding to other members of the same class, and we decided to omit this discussion here for the sake of brevity.

We have left out of the comparison the Fourier-based techniques due to several reasons. First, these methods can be implemented in different ways. If we choose the number of terms $v$ in (1) smaller than the size of the dataset, then the behavior of the truncated Fourier expansion is very similar to that of the Zernike polynomials (take note that these bases differ only in the radial coordinate). Alternatively, we can take the maximum possible size of $v$, which endows the Fourier methods with the maximum resolution capacity, but depriving them at the same time of the smoothing ability of the other modal approaches (see ${ }^{19,20,21}$ ). Last but not least, the implementation of the Fourier methods is still far from be clear and reliable, as the recent discussion ${ }^{22}$ shows.

Subject A (Fig. 4, upper left) is given analytically by

$$
C_{A}(r, q)=\min \left(\sqrt{4-r^{2}}, 1.95\right)-\sqrt{3},
$$

simulating a sphere with a cap removed by a flat cut. The main goal is to check the goodness of detection of the fast variations of the gradient.

For Subject B we use a sphere with a radial slit (Fig. 1); its level curves are represented in Fig. 5, upper left. Its analytic expression is cumbersome, and we avoid presenting it here. 

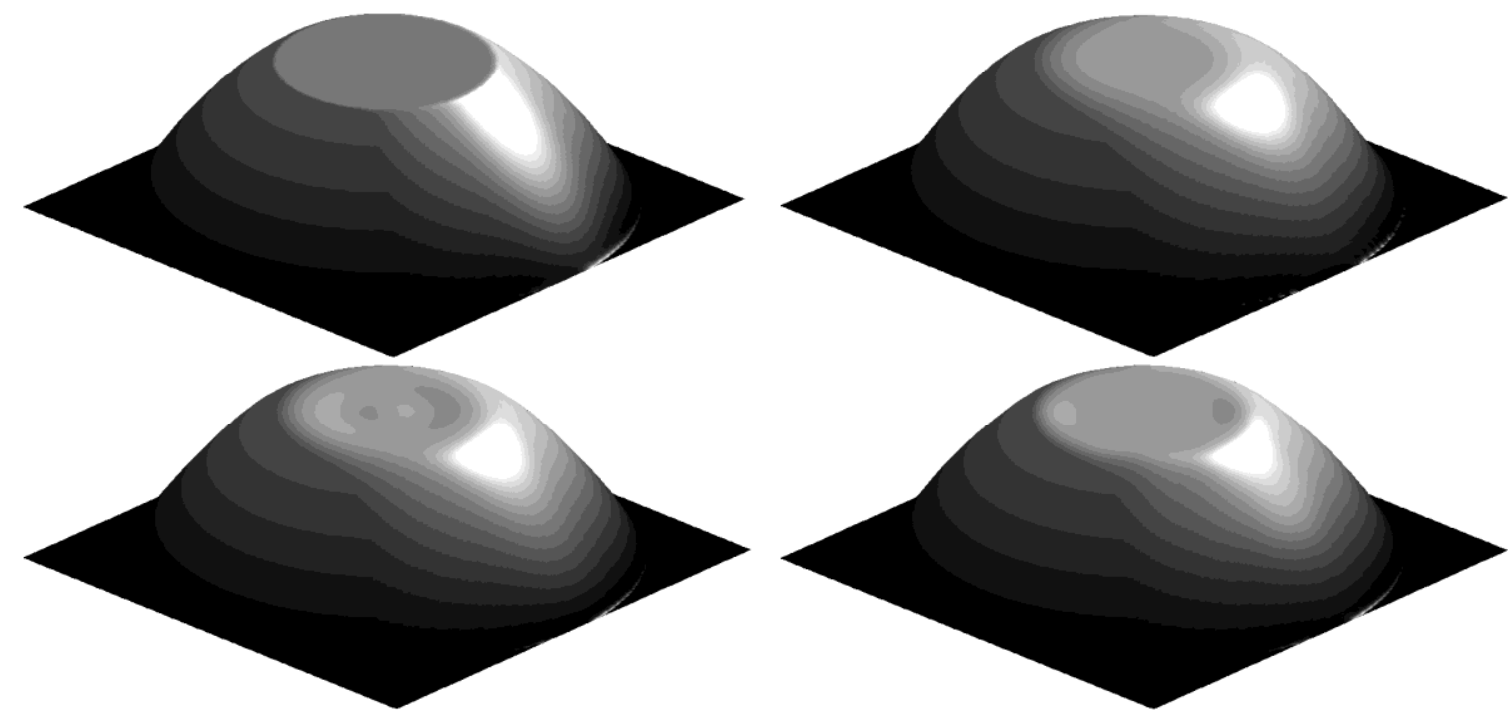

Figure 4: A 3D representation of surface A. Upper left: the original surface. Upper right: reconstruction with Zernike polynomials of radial order $6(v=28)$. Lower left: reconstruction with Bhatia-Wolf polynomials of radial order $6(v=49)$. Lower right: reconstruction with the inverse multiquadric RBFs $(\beta=-1.5, c=0.6)$ with 177 centers.

The data of Subject $\mathrm{C}$ have been collected from measurements by the CM02 Corneal Topographer (CSO, Florence, Italy) of the corneal elevations of an actual patient with keratoconus. In order to approximate the situation to the real-life scenarios, we retained the nodes where the elevations were obtained and consider a simulated keratoconus corneal surface modeled with a series of the first 136 Zernike polynomials (radial order 15). This yields an analytic expression for the surface in Fig. 6, first row, for which we already know the exact values of the corresponding Zernike coefficients. We represent it only over the domain where the reliable information is available.

We assess the quality of fit by computing in each case the mean square errors (MSE). For that purpose, after obtaining vector $\mathbf{a}$ in (2) we reconstruct the surface by formula (1) and evaluate it at the same nodes where the heights were collected. This gives us the vector of fitted elevations $\tilde{Z}$. Then

$$
M S E=\frac{1}{N}\|\tilde{Z}-\mathbf{Z}\|^{2} .
$$



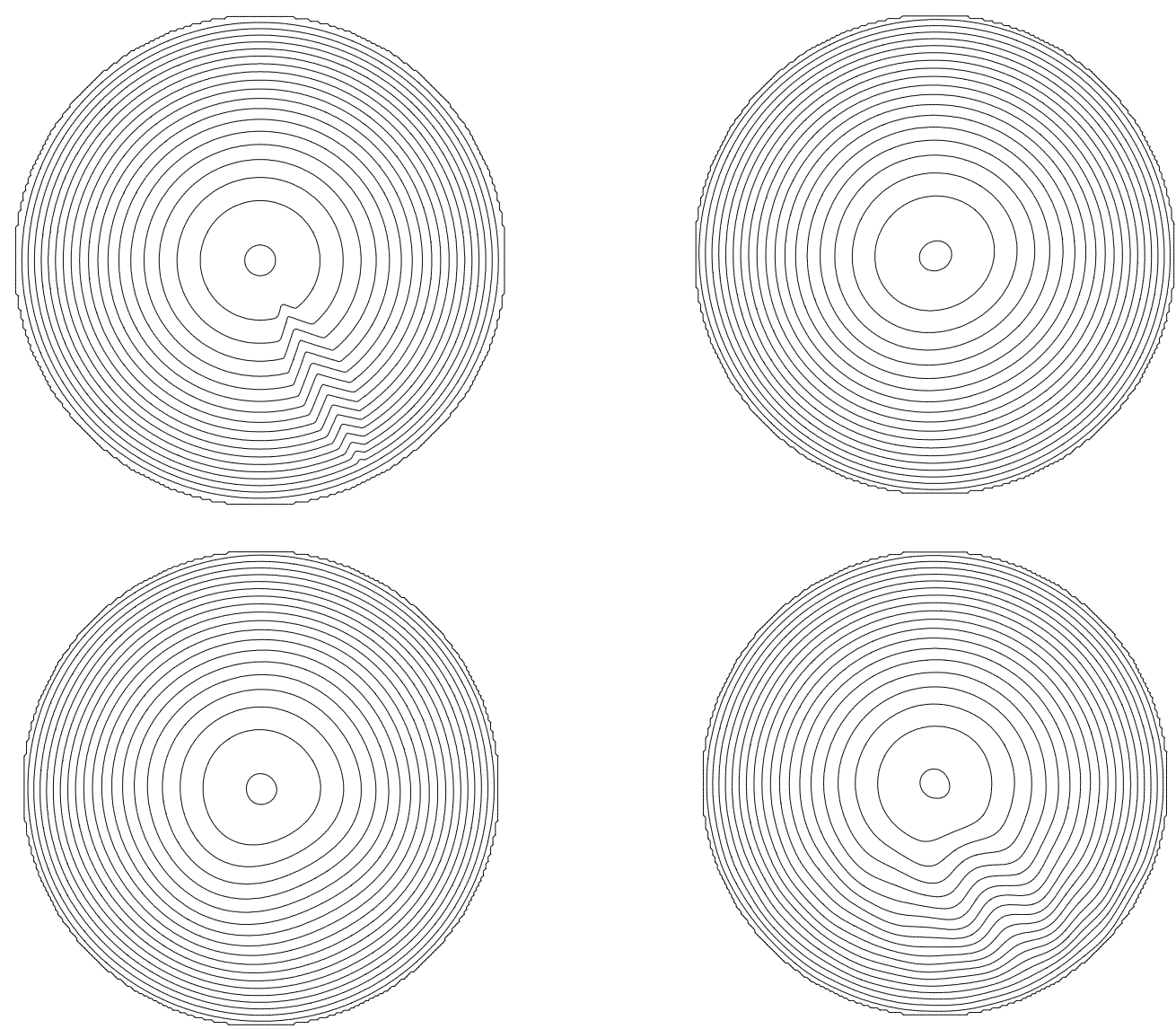

Figure 5: Contour plot for the surface B (upper left) and its reconstruction with Zernike polynomials up to order 6 (upper right) and 18, $v=190$ (lower left). Lower right: reconstruction with the inverse multiquadrics with 177 centers, using the parameters $c=1, \beta=5$.

Another criterion of the fitting quality is the accuracy in recovery of the Zernike coefficients, especially in the case of incomplete data. This issue becomes crucial for the discriminant analysis of the decease markers, or for the neural network-based diagnostic software ${ }^{4}$ such as corneal classification and condition severity grading utilities. In this sense, for Subject $\mathrm{C}$ we perform the discrete LSF with Zernike polynomials directly from the raw input data, and alternatively fitting the surface previously reconstructed by the Gaussian RBF. 

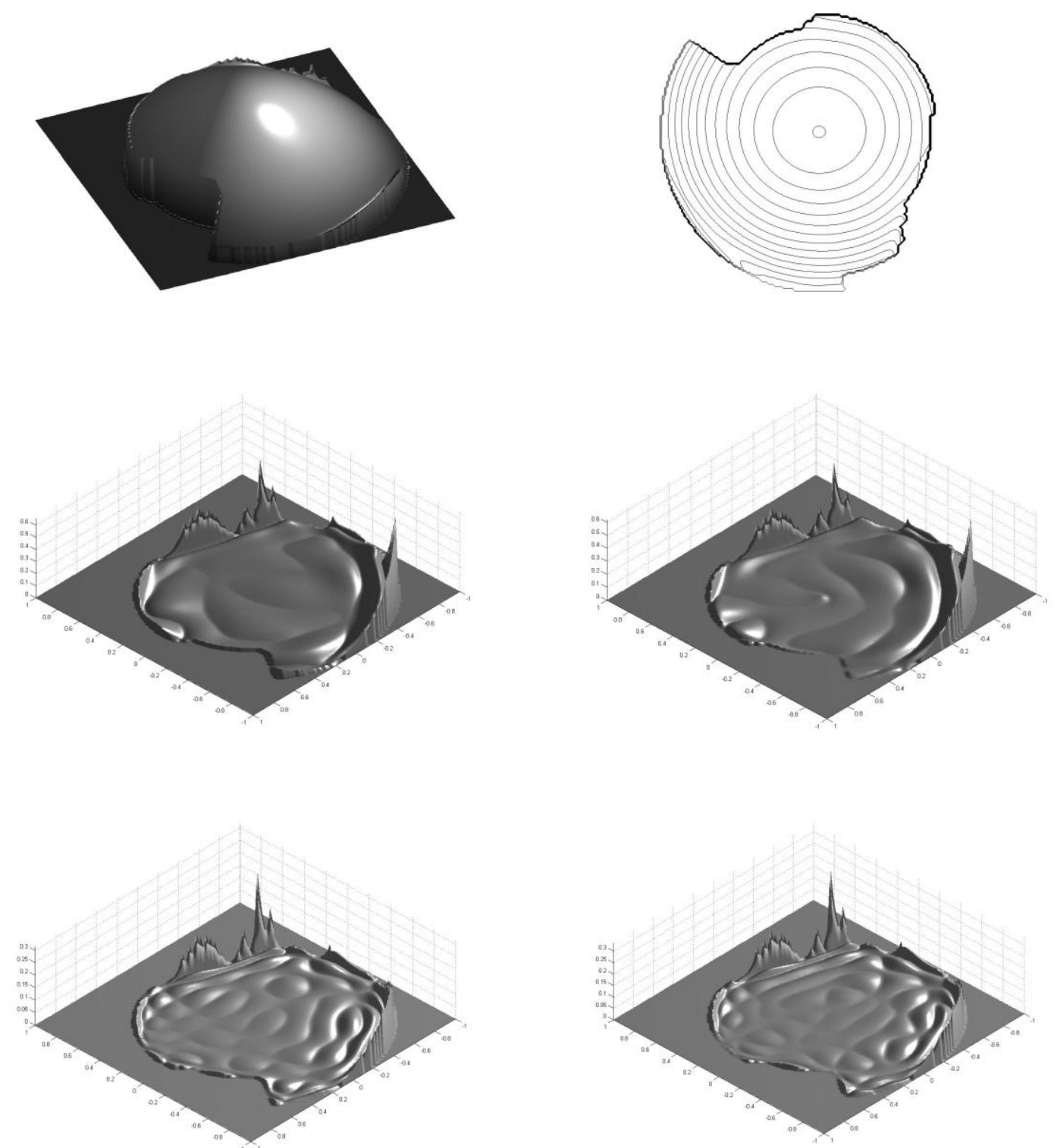

Figure 6: Contour Surface C. Up: original surface. Center: distributions of errors of the model with Zernike (left) and Bhatia-Wolf (right). Down: distributions of errors of the model with Gaussians (left) and Multiquadrics (right). plot

\section{Results}

In this section, we present a comparison of the numerical results obtained with the different methods applied to the three simulated surfaces.

There are two aspects related to the numerical side of the problem. One is the computational cost, and the other is the sensitivity of the scheme to data perturbations. 
In the former, the RBF clearly outperform the radial polynomials. The computational time is several times higher for the Zernike polynomials, even for a higly optimized vector algorithm implemented in Matlab, independently if we generate them by their recurrence relation or by the explicit formula. Nevertheless, in our implementation the reconstruction by the slowest Zernike fit with as many as 231 polynomials (radial order $\leq 20$ ) takes about 2.5 seconds (compare with ${ }^{1,3}$ ), so that execution time becomes less and less an issue with the progress of the software optimization and computing power.

On the other hand, the condition number of the collocation matrices $M$ in (2) for the RBF grows very rapidly with the dimension of the problem (number of centers). Despite this undesirable feature, they are still better fit to capture the small local variations in the shape of the cornea than the standard Zernike polynomials (see e.g. the analysis for Subject B below). This issue can be easily addressed though using a Tikhonov-type regularization combined with a SVD computation of the pseudoinverse. On the contrary, the numerical condition of the collocation matrix for Zernike polynomials initially grows slowly with the size (value of $v$ in (1)), until undersampling sets in, causing an exponential growth of the condition number (phenomenon nicely described $\mathrm{in}^{21}$, see also ${ }^{9}$ ); beyond this point an increase in the number of terms in the expansion (1) becomes counterproductive.

Let us discuss in more detail the main results in the three cases.

Surface $A$ : In the portions of the plane where the surface is smooth all approximation schemes work very well, so the error is localized in a neighborhood of the ridge formed by the cut. This explains the minimal deviation in the MSE for all methods (see Table 1). However, if our primary goal is the accurate reconstruction of the shape of the cornea, the visual analysis (Fig. 4) shows that the fitting with RBFs outperforms the fitting with radial polynomials. Clearly, the flexibility of the RBF approach, built into the scaling parameters, allows to capture more easily fast variations (or discontinuities) of the gradient of the surface.

\begin{tabular}{llll}
\hline Method & Surface A & Surface B & Surface C \\
\hline Zernike & $8.6337 \mathrm{e}-6$ & $7.0416 \mathrm{e}-7$ & $2.1226 \mathrm{e}-4$ \\
Bhatia-Wolf & $1.9262 \mathrm{e}-6$ & $6.3971 \mathrm{e}-7$ & $1.5979 \mathrm{e}-4$ \\
Gaussians & $4.6234 \mathrm{e}-7$ & $2.9088 \mathrm{e}-7$ & $8.6161 \mathrm{e}-6$ \\
Inv. Multic. & $4.5003 \mathrm{e}-7$ & $2.9042 \mathrm{e}-7$ & $8.9577 \mathrm{e}-6$ \\
\hline
\end{tabular}

Table 1. Comparison of the MSE obtained with the different methods

Surface B: the aim was to detect the relatively small details on the surface by fitting the elevation data. As it follows from Fig. 5, Zernike polynomials (and in general, radial polynomials) are less well suit for reflecting the small deformation, even if we allow unusually high orders in (1).

Surface C: in this case the deviations of the surface from an "ideal one" are more 


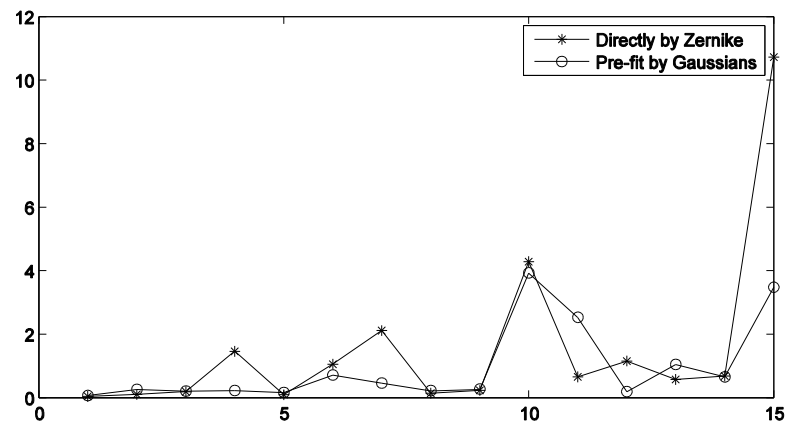

Figure 7. Relative error of the reconstruction of Zernike coefficients for Subject C directly by the LSF or fitting previously the surface with Gaussian RBF. The horizontal axis represents the 1-D index $j$ of the Zernike polynomial $Z_{j}$, while the vertical axis represents the (dimensionless) relative errors. global, and this fact is immediately reflected in the MSE in Table 1, where we gain at least two orders of precision with the RBF. However, the experiment with the reconstruction of the Zernike coefficients of the surface, either fitting it first by Gaussians or directly by Zernike polynomials, is not conclusive. Still, some coefficients are clearly better

fit with the former approach, which can be significant for an early detection of keratoconus $\left(\mathrm{see}^{28}\right)$.

These results can be improved further by using a combined approach: fitting the raw data with Zernike polynomials with a very low order $(n=1$ or $n=2)$, subtracting the fit from the original elevations, and approximating the new data by LSF with the RBF.

\section{Discussion}

The first important observation concerns the number of terms $v$ in the modal reconstruction (1). When we use radial polynomials (Zernike, Bhatia-Wolf), the number of terms corresponds to the maximum order of aberrations or frequencies that can be captured or represented by the right hand side in (1). The computational complexity of the basis functions $f_{j}$ in (1) grows with the index $j$.

Experiments show a saturation phenomenon: although for small $v$ an addition of a new term renders a substantial improvement in the goodness of the fit, higher orders have less and less impact. Moreover, a small change in a localized subset of data may imply a substantial modification of all entries of the coefficient vector $\mathbf{a}$. On the contrary, the number of terms $v$ used in the fitting with the RBF is given by the numbers of centers $Q_{j}$. Higher values of $v$ imply in this case more flexibility in the approximating scheme. The localization property of the RBF used implies also that a small local variation in the data has only a "local" impact on the coefficients of a. Basis functions $f_{j}$ in (1) for different values of the index $j$ are 
computationally identical, simply "aimed" at different points of the disk.

Hence, the amount $v$ of terms used for approximation with radial polynomials or with RBF should be compared with care.

As it was observed previously in ${ }^{14}$, Bhatia-Wolf polynomials achieve higher precision in surface approximation than their classical Zernike counterparts. Nevertheless, a clear conclusion of this research is that the Zernike polynomials still work perfectly well as a reconstruction method of a non-severely aberrated surface with a small surface regularity index (SRI). They also are an appropriate tool for recovering the lower Zernike coefficients.

However, these coefficients fail to capture small deformations of the anterior surface of the cornea. In particular, if such deformations turn out to be markers of an eye disease, it is reasonable to complement the Zernike coefficients with additional input parameters for the neural network-based diagnostic software (see the pioneering work $^{4}$, where corneal cases with no surface singularities were considered). When severe curvature changes are present, the accuracy of the fit (taking into account the small features of the surface) can become a priority, since it allows extracting reliably other shape indices of the approximated surface. In such a situation, the flexibility of the RBF functions, combining some properties of a zonal reconstruction (localization) with the simplicity of a modal scheme, can become relevant.

Thus, a combined approach seems promising: using Zernike or Bhatia-Wolf polynomials of a low degree in order to obtain the fundamental part of the shape of the cornea, with a subsequent refinement by RBF.

However, additional research is needed to address some computational issues such as an automatic selection of the scaling parameters of the RBF, or better control of the condition numbers of the corresponding collocation matrices. These aspects will be subject of a further investigation.

\section{References}

1. S. D. Klyce, M. D. Karon, M. K. Smolek, Advantages and disadvatages of the Zernike expansion for representing wave aberration of the normal and aberrated eye, J. Refractive Surgery 20 (2004) S537-S541.

2. M. K. Smolek, S. D. Klyce, Zernike polynomial fitting fails to represent all visually significant corneal aberrations, Invest. Ophthalmol. Vis. Sci. 44 (11) (2003) 46764681.

3. L. A. Carvalho, Accuracy of Zernike Polynomials in Characterizing Optical 
Aberrations and the Corneal Surface of the Eye, Invest. Ophthalmol. Vis. Sci. 46 (2005), 1915-1926.

4. L. A. Carvalho, Preliminary Results of Neural Networks and Zernike Polynomials for Classification of Videokeratography Maps, Optometry and Vision Science 82 (2) (2005), 151-158.

5. M. K. Smolek, S. D. Klyce, and D. S. Brenner, Zernike polynomialterms and corneal indices as neural network inputs for videokeratography classification, Invest. Ophthalmol. Vis. Sci. 38 (1997), S920.

6. J. T. Schwiegerling, J. E. Greivenkamp, Keratoconus detection based on videokeratoscopic height data, Optometry and Vision Science 73 (1989) 721-728.

7. P. Artal, E. Berrio, A. Guirao, P. Piers, Contribution of the cornea and internal surfaces to the change of ocular aberrations with age, J. Opt. Soc. Am. A 19 (2002) 137-143.

8. M. K. Smolek, S. D. Klyce, Goodness-of-prediction of Zernike polynomial fitting to corneal surfaces, J. Cataract Refract. Surg. 31 (2005) 2350-2355.

9. R. Iskander, M. J. Collins, B. Davis, Optimal modeling of corneal surfaces with Zernike polynomials, IEEE Trans. Biomed. Eng. 48 (1) (2001) 87-95.

10. M. Ares, S. Royo, Comparison of cubic B-spline and Zernike-fitting techniques in complex wavefront reconstruction, Applied Optics 45 (2006) 6945-6964.

11. L. Thibos, R. A. Applegate, J. T. Schwiegerling, and R. Webb, Standards for reporting the optical aberrations of eyes, in Vision Science and its Applications, OSA Technical Digest (Optical Society of America, 2000), paper SuC1.

12. J. J. Rozema, D. E. M. Van Dyck, M.-J. Tassignon, Clinical comparison of 6 aberrometers. Part 1: technical specifications, J. Cataract Refract. Surg. 31 (2005) 1114-1127.

13. A. B. Bhatia, E. Wolf, On the circle polynomials of Zernike and related orthogonal sets, Proc. Cambridge Philos. Soc. 50 (1954) 40-53.

14. R. Iskander, M. R. Morelande, M. J. Collins, B. Davis, Modeling of corneal surfaces with radial polynomials, IEEE Trans. Biomed. Eng. 49 (4) (2002) 320-328.

15. D. R. Myrick, A generalization of the radial polynomials of F. Zernike, J. SIAM Appl. Math. 14 (3) (1966) 476-489.

16. Y. Xu, A family of Sobolev orthogonal polynomials on the unit ball, J. Approx. Theory 138 (2006) 232-241.

17. Y. Xu, Sobolev orthogonal polynomials defined via gradient on the unit ball, J. Approx. Theory 152 (2008) 52-65.

18. F. Roddier, C. Roddier, Wavefront reconstruction using iterative Fourier transform, Applied Optics 30 (1991) 501-503. 
19. G. Dai, Comparison of wavefront reconstruction with Zernike polynomials and Fourier coefficients, Journal of Refractive Surgery 22 (11) (2006) 943-948.

20. L. Wang, D. Chernyak, D. Yeh, D. D. Koch, Fitting behavior of Fourier transform and Zernike polynomials, J. Cataract Refract. Surg. 33 (2007) 999-1004.

21. G. Yoon, S. Pantanelli, S. MacRae, Comparison of Zernike and Fourier wavefront reconstruction algorithms in representing corneal aberration of normal and abnormal eyes, J. Refractive Surgery 24 (2008) 582-590.

22. "Wavefront Reconstruction Methods", Letters to the Editor, J. Refractive Surgery 25 (2009) 9-11.

23. H. Wendland, Scattered Data Approximation, Vol. 17 of Cambridge Monographs on Applied and Computational Mathematics, Cambridge Univ. Press, Cambridge, UK, 2005.

24. M. D. Buhmann, Radial Basis Functions: Theory and Implementations, Cambridge Univ. Press, 2003.

25. M. Montoya-Hernández, M. Servín, D. Malacara-Hernández, G. Paez, Wavefront fitting using Gaussian functions, Optics Communications 163 (1999) 259-269.

26. L. N. Trefethen, D. Bau III, Numerical Linear Algebra, SIAM, 1997.

27. D. Alonso-Caneiro, R. Iskander, M. J. Collins, Estimating corneal surface topography in videokeratoscopy in the presence of strong signal interference, IEEE Trans. Biomed. Eng. 55 (10) (2008) 2381-2387.

28. J. L. Alió, M. H. Shabayek, Corneal higher order aberrations: a method to grade keratoconus, Journal of Refractive Surgery 22 (2006) 539-545. 